\section{Commentary: Amiodarone and anticoagulation in postoperative atrial fibrillation: Less is more?}

\author{
Derrick Y. Tam, MD, and \\ Stephen E. Fremes, MD, MSc
}

New-onset postoperative atrial fibrillation (POAF) affects $25 \%$ to $50 \%$ of patients after cardiac surgery and has been shown to be associated with increased morbidity and mortality. The practice of treating POAF remains variable with 2 Class I and 5 Class IIa recommendations in the 2014 American College of Cardiology/American Heart Association guidelines. ${ }^{1}$ Briefly summarized, the guidelines recommend the use of beta-blockers or calcium channel blockers (Class I, level of evidence [LOE]: A and B, respectively) or amiodarone (Class IIa, LOE A) with the addition of oral anticoagulation (Class IIa, LOE: C) for those who are not in sinus rhythm for POAF. However, whether these guidelines are strictly adhered to in the real-world realm of cardiac surgery remains unknown.

Matos and colleagues ${ }^{2}$ investigated the use of oral anticoagulation and amiodarone after isolated coronary artery bypass grafting in patients with new-onset POAF in the Society of Thoracic Surgeons Adult Cardiac Surgery Database. Examining 166,747 patients from 2011 to 2018, the authors found that only one-quarter received an oral anticoagulant, of whom the majority (four-fifths) received amiodarone. In the three-quarters of patients who did not receive an oral anticoagulant, again, approximately threequarters received amiodarone. Approximately one-third of patients who received an oral anticoagulant were prescribed a direct oral anticoagulant. There were some interesting findings in the outcomes from this analysis after adjusting for patient baseline characteristics. Although the risk of readmission for bleeding was higher in those receiving an oral anticoagulant, there was no difference in the risk of stroke

\footnotetext{
From the Division of Cardiac Surgery, Schulich Heart Centre, Department of Surgery, Sunnybrook Health Sciences Centre, University of Toronto, Toronto, Ontario, Canada.

Disclosures: Authors have nothing to disclose with regard to commercial support.

Received for publication Feb 4, 2020; accepted for publication Feb 6, 2020; available ahead of print Feb 14, 2020.

Address for reprints: Stephen E. Fremes, MD, MSc, Schulich Heart Centre, Sunnybrook Health Sciences Centre, 2075 Bayview Ave, Room H4 05, Toronto, Ontario M4N 3M5, Canada (E-mail: Stephen.fremes@sunnybrook.ca).

J Thorac Cardiovasc Surg 2021;162:625-6

$0022-5223 / \$ 36.00$

Copyright (C) 2020 by The American Association for Thoracic Surgery

https://doi.org/10.1016/j.jtcvs.2020.02.022
}

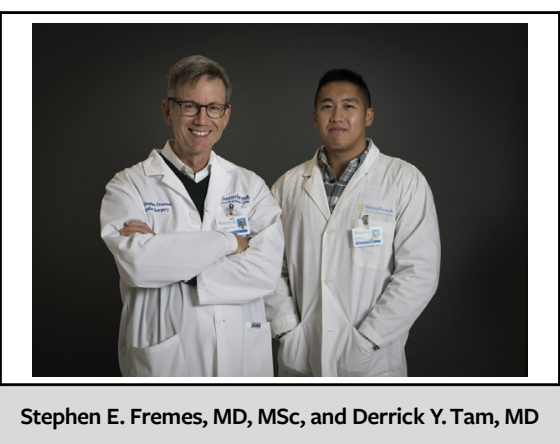

\section{CENTRAL MESSAGE \\ The optimal management of POAF \\ is unclear, and treatment remains \\ highly variable in North America.}

readmission at 30 days with rhythm control alone, although those who received both an anticoagulant and amiodarone had less stroke readmissions at 30 days. Finally, there was an increase in mortality seen with those on anticoagulant, with more deaths in those prescribed a direct oral anticoagulant rather than those on warfarin. The cause for this increase in death was not entirely clear because readmission for stroke and bleeding was similar between those who received a direct oral anticoagulant versus warfarin.

Nonetheless, this analysis must be interpreted in the context of significant limitations. First, the Society of Thoracic Surgeons definition of POAF is either 1 hour of atrial fibrillation or treatment for atrial fibrillation, and this may be overly sensitive. As such, many of these patients with atrial fibrillation may have had paroxysmal atrial fibrillation that did not necessitate treatment. Furthermore, these findings are subject to treatment allocation bias; the exact clinical indications for the prescription of amiodarone or oral anticoagulants was not known because of a lack of granularity in the dataset. Although additional information was gleaned by authors through examination of the CHADS2-Vasc score (a measure of stroke risk in patients with atrial fibrillation), we note that this score was not designed for patients with POAF. Of note, there was not enough information to calculate a HAS-BLED score to determine the patient's risk of bleeding to weigh against his/her risk for thromboembolism. ${ }^{3}$ Although amiodarone use was recorded, there was no additional information regarding the use of other classes of antiarrhythmics that may be used in the treatment of atrial fibrillation. Nonetheless, the authors did examine beta-blocker use and found that overall use of preoperative and postoperative betablockers was high in this study. Rhythm versus rate control for POAF was studied in a large, randomized clinical trial 
that showed no difference between the treatment arms. ${ }^{4}$ Along this vein, it is not clear whether amiodarone was used as a prophylactic drug or used as treatment for atrial fibrillation. Finally, there remains a paucity of information regarding the long-term outcomes of these patients in this study. Although most POAF cases resolve within 7 days, there is a long-term increased risk of stroke and bleeding in those with persistent atrial fibrillation beyond 30 days that is not captured in this analysis. Further information regarding stroke and bleeding readmission by low-, medium-, and high-risk CHADS2-Vasc score would have been informative.

Despite these limitations, the authors should be congratulated on this important study because it sheds light on the treatment of a common complication of cardiac surgery. The fact that the authors found significant geographical variation in North America on the treatment of POAF suggests that treatment may be independent of patient factors and affected by institution and geographical practice patterns. These findings suggest that there remains a need to better delineate the role of anticoagulation in patients with POAF, and this is currently being investigated in the Anticoagulation for New-Onset Post-Operative Atrial Fibrillation After CABG (PACES) trial (NCT04045665) of oral anticoagulant to antiplatelet therapy for the management of POAF. In the PACES trial, patients may receive warfarin or a direct oral anticoagulant. Although the direct oral anticoagulants have a faster onset of action and do not require titration compared with warfarin, concerns regarding bleeding and lack of availability of reversal agents for all direct oral anticoagulants may limit their popularity among surgeons concerned about postoperative bleeding. Thus, while we await the completion and reporting of the PACES trial, less may be more in the management of POAF.

\section{References}

1. January CT, Wann LS, Alpert JS, Calkins H, Cigarroa JE, Cleveland JC Jr, et al. 2014 AHA/ACC/HRS Guideline for the Management of Patients With Atrial Fibrillation: A Report of the American College of Cardiology/American Heart Association Task Force on Practice Guidelines and the Heart Rhythm Society. Circulation. 2014;130:e199-267.

2. Matos JD, McIlvaine S, Grau-Sepulveda M, Jawitz OK, Brennan JM, Khabbaz KR, et al. Anticoagulation and amiodarone for newatrial fibrillation after coronary artery bypass grafting: prescription patterns and 30-day outcomes in the United States and Canada. J Thorac Cardiovasc Surg. 2021;162:616-24.e3.

3. Roldán V, Marín F, Manzano-Fernández S, Gallego P, Vílchez JA, Valdés M, et al. The HAS-BLED score has better prediction accuracy for major bleeding than CHADS2 or CHA2DS2-VASc scores in anticoagulated patients with atrial fibrillation. J Am Coll Cardiol. 2013;62:2199-204.

4. Gillinov AM, Bagiella E, Moskowitz AJ, Raiten JM, Groh MA, Bowdish ME, et al. Rate control versus rhythm control for atrial fibrillation after cardiac surgery. N Engl J Med. 2016;374:1911-21.

\section{Commentary: A step toward solving a stubborn problem... maybe}

Nathaniel B. Langer, MD, MSc, and

Gus J. Vlahakes, MD

In this issue of the Journal, Matos and colleagues ${ }^{1}$ report the prescribing patterns of anticoagulation and amiodarone in patients with new-onset atrial fibrillation (AF) after

\footnotetext{
From the Department of Surgery, Harvard Medical School; and Division of Cardiac Surgery, Massachusetts General Hospital, Boston, Mass.

Disclosures: Authors have nothing to disclose with regard to commercial support.

Received for publication Feb 6, 2020; revisions received Feb 6, 2020; accepted for publication Feb 11, 2020; available ahead of print Feb 19, 2020.

Address for reprints: Gus J. Vlahakes, MD, Division of Cardiac Surgery, Massachusetts General Hospital, 55 Fruit St, Cox 630, Boston, MA 02114 (E-mail: vlahakes. gus@mgh.harvard.edu).

J Thorac Cardiovasc Surg 2021;162:626-7

$0022-5223 / \$ 36.00$

Copyright (c) 2020 by The American Association for Thoracic Surgery

https://doi.org/10.1016/j.jtcvs.2020.02.041
}

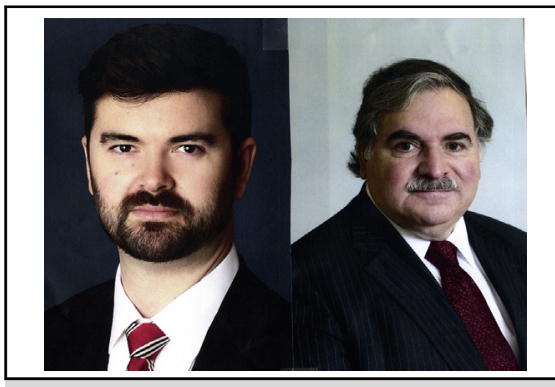

Nathaniel B. Langer, MD, MSc (left), and Gus J. Vlahakes, MD (right)

CENTRAL MESSAGE

Ideal management of atrial fibril-

lation after coronary artery

bypass grafting remains elusive, and actual national practice pat-

terns deviate from society

guidelines. 